\title{
Peptic ulcer disease and non-steroidal anti-inflammatory drugs
}

\section{SUMMARY}

Non-steroidal anti-inflammatory drugs including low-dose aspirin are some of the most commonly used medicines. They are associated with gastrointestinal mucosal injury.

Before prescribing, it is important to assess the patient's gastrointestinal risk factors such as age and history of peptic ulcers. Patients at high risk may require co-prescription to reduce the risk of peptic ulcers.

A daily dose of a proton pump inhibitor is the most effective method of reducing the risk of ulcers induced by non-steroidal anti-inflammatory drugs.

\section{Introduction}

A peptic ulcer is a defect in the upper gastrointestinal mucosa that extends through the muscularis mucosa into deeper layers of the gut wall. There are two major risk factors for peptic ulcer disease - Helicobacter pylori and non-steroidal anti-inflammatory drugs (NSAIDs). NSAIDs including low-dose aspirin are some of the most commonly used drugs. They have good efficacy and a long history of clinical use, but can cause peptic ulcers which may have fatal complications.' Given widespread use of NSAIDs and aspirin, the associated gastrointestinal toxicities have substantial implications for the healthcare system. ${ }^{2}$

\section{Mechanism of action}

The therapeutic effects of NSAIDs are mediated by their inhibition of prostanoid biosynthesis. ${ }^{3}$ Prostanoid derivatives arise from the conversion of arachidonic acid by cyclo-oxygenase (COX) isoenzymes following cell injury.

There are two distinct isoforms of COX. COX-1 is present in the majority of cells including endothelial cells, gastrointestinal epithelium and platelets, and functions continuously. In contrast COX-2 is present in only a few tissues and is induced by inflammation. NSAIDs exert their therapeutic anti-inflammatory and analgesic effects by inhibiting COX-2. The gastric and renal toxicities of the drugs are related to inhibition of the COX-1 isoform. ${ }^{4,5}$ There is a spectrum of COX-1 and COX-2 inhibition across the class of NSAIDs.

\section{Ulcers and NSAIDs}

Peptic ulcer disease is a well-recognised complication of NSAID use. Inhibition of COX-1 in the gastrointestinal tract leads to a reduction of prostaglandin secretion and its cytoprotective effects in gastric mucosa. This therefore increases the susceptibility to mucosal injury. ${ }^{6}$ Inhibition of COX-2 may also play a role in mucosal injury.

\section{Risk factors}

Gastrointestinal toxicity with NSAIDs, including low-dose aspirin, is highest in patients with risk factors. These include increased age (>65 years), past history of peptic ulcer disease, heart disease, and co-prescription of antiplatelets, corticosteroids and anticoagulants. In addition, using higher doses of NSAIDs leads to an increased risk of upper gastrointestinal complications. ${ }^{7}$ Prolonged NSAID use and $H$. pylori infection are also associated with an increased risk of gastrointestinal toxicity.

In patients who are chronic users of NSAIDs and who have no risk factors, only $0.4 \%$ have serious adverse events. However, the risk is as high as $9 \%$ in patients with multiple risk factors. ${ }^{8}$ Before prescribing for a patient with risk factors always consider if there are alternatives to NSAIDs.

\section{Which NSAID to use?}

All NSAIDs cause some degree of gastrointestinal toxicity. Large pooled data from placebo-controlled trials show that all evaluated NSAIDs including COX-2 inhibitors, diclofenac, ibuprofen and naproxen were associated with an increased risk of gastrointestinal injury. ${ }^{9}$ However, this risk varies between the drugs. The relative risk of upper gastrointestinal complications for aceclofenac, celecoxib and ibuprofen is low (<2). Diclofenac, meloxicam and ketoprofen are intermediate (2-4) while naproxen, indomethacin and diflunisal have a higher relative risk (4-5). The highest pooled relative risk is associated with piroxicam (7.4) and ketorolac (11.5). ${ }^{7}$

\section{Musa Drini}

Associate lecturer Medical School Australian National University

Staff specialist Canberra Hospital Gastroenterologist gastrotrACT

Canberra

\section{Keywords}

misoprostol, non-steroidal anti-inflammatory drugs (NSAIDs), peptic ulcers, proton pump inhibitors

Aust Prescr 2017:40:91-3 http://dx.doi.org/10.18773/ austprescr.2017.037

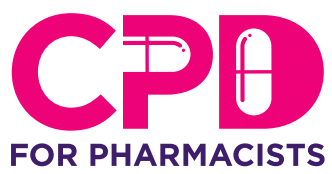

This article has a continuing professional development activity for pharmacists available at http://learn.nps.org.au 
Drugs with greater selectivity for COX-2 than COX-1 should have less gastrointestinal toxicity. Large pooled data showed that the predicted absolute annual risk of upper gastrointestinal complications was lower for COX-2 inhibitors than naproxen and ibuprofen. ${ }^{7}$ However, COX-2 inhibitors are associated with an increased risk of cardiovascular events. There is little evidence of an increased risk of cardiovascular complications with use of a low dose of diclofenac. However, to avoid possible cardiovascular complications the use of NSAIDs should be at the lowest possible dose and for the shortest time.

\section{Low-dose aspirin}

Low-dose aspirin is widely prescribed to reduce cardiovascular and cerebrovascular thromboembolic events. Patients who use low-dose aspirin tend to be older and likely to use concurrent antiplatelet or anticoagulant drugs that would lead to an increased risk of gastrointestinal toxicity. It has been shown that the relative risk for gastrointestinal toxicity such as bleeding is as high as 4 in high-risk patients. Meta-analysis of randomised controlled trials of 57000 patients taking low-dose aspirin revealed a relative risk of 2 for significant gastrointestinal adverse events such as bleeding. ${ }^{10}$ In patients with a history of ulcer bleeding consideration needs to be given to secondary ulcer prevention with proton pump inhibitor therapy. Changing aspirin to other antiplatelet therapy is not recommended.

\section{Strategies to reduce gastrointestinal toxicity}

Caution is needed to minimise the known adverse effects. Before prescribing, the medical history needs to be reviewed in order to assess the risk of gastrointestinal toxicity. This includes asking about the use of over-the-counter aspirin and NSAIDs. Consideration needs to be given to the choice of NSAID and the duration of therapy. Patients need to be informed and be aware of potential serious adverse events.

\section{Proton pump inhibitors}

Proton pump inhibitors reduce the secretion of gastric acid by inhibiting $\mathrm{H}^{+} / \mathrm{K}^{+}$-ATPase. They are widely used to induce healing of gastric and duodenal ulcers. In a double-blind randomised trial, a standard dose of proton pump inhibitor therapy was efficacious in ulcer healing and preventing recurrence of gastroduodenal injury in patients taking NSAIDs. " Proton pump inhibitors are slightly better at preventing duodenal ulcers than gastric ulcers. ${ }^{12}$

A recent meta-analysis assessed current strategies for the prevention of gastrointestinal toxicity. It found that selective COX-2 inhibitors in combination with proton pump inhibitors were associated with the lowest absolute event probability for ulcer complications (0.07; 95\% confidence interval 0.02-0.18). ${ }^{13}$ This strategy may be the most effective way to reduce the risk of ulcer complications, followed by the use of a selective COX-2 inhibitor alone and a non-selective NSAID combined with a proton pump inhibitor. Importantly all three strategies were well tolerated by patients. Due to their acceptable adverse effect profile, proton pump inhibitors have assumed therapeutic dominance for minimising the gastrointestinal risks of NSAIDs.

\section{$\mathrm{H}_{2}$-receptor antagonists}

$\mathrm{H}_{2}$-receptor antagonists have an adverse effect profile that is comparable to proton pump inhibitor therapy. However, the pooled data analysis showed that a single daily dose does not protect against NSAID-related gastric ulcers, but double doses of $\mathrm{H}_{2}$-receptor antagonist (at least ranitidine $300 \mathrm{mg}$ twice daily) were effective in preventing gastric and duodenal ulcers when compared to placebo. ${ }^{14}$

\section{Misoprostol}

Misoprostol is an analogue of prostaglandin $E_{1}$. Its gastro-protective effect is achieved by inhibition of gastric acid and pepsin release and it improves the resistance of the gastric mucosa. This effect is largely dose dependent. A large pooled data analysis showed misoprostol was effective in preventing gastric (74\%) and duodenal ulcers (53\%) in comparison with placebo. ${ }^{10}$ Unfortunately, the clinical usefulness of the misoprostol is limited largely by its gastrointestinal adverse effects such as cramping, abdominal pain and diarrhoea, making it a third-line drug option.

\section{Helicobacter pylori and NSAIDs in peptic ulcer disease}

Testing for $H$. pylori for ulcer prevention in asymptomatic patients needs to be assessed on a case-by-case basis and is not routinely recommended. However, testing and eradicating $H$. pylori is recommended in patients with a past history of peptic ulcer disease before starting low-dose aspirin or NSAIDs. ${ }^{15}$

\section{Conclusion}

Chronic use of NSAIDs including low-dose aspirin is associated with gastrointestinal mucosal injury. However, major adverse events are relatively infrequent. Patients with multiple risk factors such as a previous history of peptic ulcer disease, increasing age, co-prescription of corticosteroids and anticoagulants, and high-dose and long-term use of NSAIDs are at the highest risk of major gastrointestinal toxicity. In patients with multiple risk factors, physicians need 
to assess these risks before prescribing NSAIDs and adopt risk-minimising strategies.

Simple measures such as using the lowest dose for short periods of time when possible will prevent some of the NSAID-related toxicity. Selective COX-2 inhibitors also will reduce gastrointestinal adverse events when compared to non-selective NSAIDs. Proton pump inhibitor therapy is efficacious and has an acceptable adverse effect profile in comparison with misoprostol.

\section{REFERENCES}

1. Atchison JW, Herndon CM, Rusie E. NSAIDs for musculoskeletal pain management: current perspectives and novel strategies to improve safety. J Manag Care Pharm 2013;19(Suppl A):1-19. http://dx.doi.org/10.18553/jmcp.2013.19.s9.1

2. Smalley WE, Griffin MR, Fought RL, Ray WA. Excess costs from gastrointestinal disease associated with nonsteroidal anti-inflammatory drugs. J Gen Intern Med 1996;11:461-9. http://dx.doi.org/10.1007/BF02599040

3. Ricciotti E, FitzGerald GA. Prostaglandins and inflammation. Arterioscler Thromb Vasc Biol 2011;31:986-1000 http://dx.doi.org/10.1161/ATVBAHA.110.207449

4. Dvornik DM. Tissue selective inhibition of prostaglandin biosynthesis by etodolac. J Rheumatol Suppl 1997;47:40-7.

5. Robinson DR. Regulation of prostaglandin synthesis by antiinflammatory drugs. J Rheumatol Suppl 1997;47:32-9.

6. Brune K, Patrignani P. New insights into the use of currently available non-steroidal anti-inflammatory drugs. J Pain Res 2015;8:105-18. http://dx.doi.org/10.2147/JPR.S75160

7. Castellsague J, Riera-Guardia N, Calingaert B, Varas-Lorenzo C, Fourrier-Reglat A, Nicotra F, et al. Individual NSAIDs and upper gastrointestinal complications. Drug Saf 2012;35:1127. http://dx.doi.org/10.1007/BF03261999

8. Coxib and traditional NSAID Trialists' (CNT) Collaboration, Bhala N, Emberson J, Merhi A, Abramson S, Arber N, et al. Vascular and upper gastrointestinal effects of non-steroidal anti-inflammatory drugs: meta-analyses of individual participant data from randomised trials. Lancet 2013;382:769-79. http://dx.doi.org/10.1016/ S0140-6736(13)60900-9

9. Silverstein FE, Graham DY, Senior JR, Davies HW, Struthers BJ, Bittman RM, et al. Misoprostol reduces serious gastrointestinal complications in patients with rheumatoid arthritis receiving nonsteroidal anti-inflammatory drugs. A randomized, double-blind, placebo-controlled trial. Ann Intern Med 1995;123:241-9. http://dx.doi.org/10.7326/ 0003-4819-123-4-199508150-00001
Before prescribing an NSAID, prophylactic proton pump inhibitor therapy needs to be offered to patients with a past history of peptic ulcer disease and those on dual antiplatelet therapy or anticoagulant therapy. $<$

\section{Conflict of interest: none declared}

10. McQuaid KR, Laine L. Systematic review and meta-analysis of adverse events of low-dose aspirin and clopidogrel in randomized controlled trials. Am J Med 2006;119:624-38. http://dx.doi.org/10.1016/j.amjmed.2005.10.039

11. Hawkey CJ, Karrasch JA, Szczepañski L, Walker DG, Barkun A, Swannell AJ, et al. Omeprazole compared with misoprostol for ulcers associated with nonsteroidal antiinflammatory drugs. Omeprazole versus misoprostol for NSAID-induced ulcer management (OMNIUM) study group. N Engl J Med 1998;338:727-34. http://dx.doi.org/10.1056/ NEJM199803123381105

12. Cullen D, Bardhan KD, Eisner M, Kogut DG, Peacock RA Thomson JM, et al. Primary gastroduodenal prophylaxis with omeprazole for non-steroidal anti-inflammatory drug users. Aliment Pharmacol Ther 1998;12:135-40.

13. Yuan JQ, Tsoi KK, Yang M, Wang JY, Threapleton DE, Yang ZY, et al. Systematic review with network metaanalysis: comparative effectiveness and safety of strategies for preventing NSAID-associated gastrointestinal toxicity. Aliment Pharmacol Ther 2016;43:1262-75. http://dx.doi.org/ 10.1111/apt.13642

14. Rostom A, Dube C, Wells G, Tugwell P, Welch V, Jolicoeur E, et al. Prevention of NSAID-induced gastroduodenal ulcers. Cochrane Database Syst Rev 2002;4:CD002296. http://dx.doi.org/10.1002/14651858.CD002296

15. Klitz U, Zochling J, Schmidt WE, Braun J. Use of NSAIDs and infection with Helicobacter pylori - what does the rheumatologist need to know? Rheumatology (Oxford) 2008;47:1342-7. http://dx.doi.org/10.1093/rheumatology/ ken123

\section{SELF-TEST} QUESTIONS

True or false?

1. The most effective method to prevent peptic ulcers induced by non-steroidal antiinflammatory drugs is to co-prescribe an $\mathrm{H}_{2}$-antogonist.

2. Proton pump inhibitors are more effective in preventing duodenal, rather than gastric ulcers, induced by non-steroidal antiinflammatory drugs.

Answers on page 119 